\title{
Neutropenic enterocolitis due to Clostridium septicum infection
}

\author{
ASHLEY KING, ${ }^{*}$ ANITA RAMPLING,$\dagger$ DGD WIGHT, ${ }^{*}$ RE WARREN $\dagger$ \\ Departments of *Histopathology and $\nmid$ Medical Microbiology, Addenbrooke's Hospital, Cambridge
}

SUMMARY Three cases of neutropenic enterocolitis are described. This unusual condition occurs almost exclusively in neutropenic patients and has a fulminating course which is almost invariably fatal without surgical intervention. The lesion is centred on the caecum and hitherto its pathogenesis has been unclear, partly because most studies have been performed on material obtained at necropsy. In these three patients, all of whom were treated surgically, Clostridium septicum was identified by specific immunofluorescence in the bowel wall of the resected specimens. Two patients also had $C$ septicum septicaemia. Various forms of mucosal damage can be identified which predispose towards invasion of the bowel wall by this organism. These cases provide further confirmation of a primary role for $C$ septicum in the pathogenesis of neutropenic enterocolitis.

Several types of gastrointestinal lesion have been described in patients with leukaemia. ${ }^{\prime}$ These include leukaemic infiltration, mucosal haemorrhage, fungal infection, and atypical epithelial changes directly attributable to cytotoxic drugs. ${ }^{2}$ More recently, necrotising enterocolitis, associated with localised infection or bacteraemia, or both, has been increasingly recognised as a particular complication of leukaemia. $^{3}$

The name necrotising enterocolitis is confusing and probably includes three separate and distinct entities: pseudomembranous colitis, ischaemic colitis, and neutropenic enterocolitis. ${ }^{4}$ Pseudomembranous colitis has a characteristic morphology and is linked with antibiotic treatment, and Clostridium difficile or its toxin or both is present in the stool. Acute ischaemic colitis is usually associated with ulceration, extensive mucosal and submucosal haemorrhage, and fibrin thrombi in vessels. The third group variously known as the ileocaecal syndrome, typhlitis, or, most commonly, neutropenic enterocolitis $^{5-7}$ is a distinct clinicopathological syndrome of unknown aetiology in which patients often present with abdominal pain and fever resembling appendicitis. The lesion, often diagnosed only at necropsy, is usually centred on the caecum, which shows pronounced thickening of its wall with intense

Accepted for publication 16 November 1983 oedema and necrosis and variable numbers of microorganisms. Although the mortality is high, there have been recent reports of successful treatment by surgery ${ }^{8-10}$ and even antibiotics alone. ${ }^{11}$

The pathogenesis of neutropenic enterocolitis is not entirely clear. The link with neutropenia seems strong since the condition is also encountered in neutropenia attributable to other causes-for example, cyclic neutropenia ${ }^{12-15}$ and drug induced agranulocytosis. ${ }^{16}$

Although there are few detailed descriptions of the pathology, most authors have seen organisms within the lesions. The organisms cultured from blood or bowel have, however, been varied and often mixed, and, as most descriptions refer to postmortem material, it is not always clear whether the organisms have played a primary role or are merely secondary invaders. There have been three recent reports of four cases of neutropenic colitis ${ }^{12-14}$ in which $C$ septicum septicaemia was associated with characteristic ileocaecal disease. In three of these cases Gram positive rods were seen in the bowel wall. Rifkin postulated that this organism might be crucial to the development of neutropenic enterocolitis. ${ }^{13}$ The many other organisms found by other authors may then be secondary invaders or even represent postmortem growth.

$C$ septicum is not a common cause of nontraumatic sepsis in man. Most of the recent reports of $C$ septicum infection, however, have been in 
patients with leukaemia or colonic cancer. ${ }^{17-19}$ Although none of these authors gives a detailed description of the pathological findings, most authors describe haemorrhage and necrosis of the caecum or distal ileum or "caecitis."

In this report we describe three leukaemic patients with classic neutropenic enterocolitis, each of whom was subjected to bowel resection. In all three patients material for histological examination was available early in the course of the patient's illness and there was therefore little or no opportunity for secondary bacterial invasion. In all three cases $C$ septicum bacilli alone were shown in the bowel wall by specific immunofluorescence.

\section{Material and methods}

C septicum fluorescein isothiocyanate labelled " $O$ " antiserum was used, which is a mixture of three different rabbit antisera raised against three strains: groups I and II described by Moussa ${ }^{20}$ and a third serologically distinct strain isolated in Tasmania (donated by Wellcome Research Laboratories).

$C$ difficile fluorescein isothiocyanate labelled " $\mathrm{O}$ " antiserum was raised in rabbits against an $18 \mathrm{~h}$ culture of a toxigenic strain of $C$ difficile (CLN 34854/ 82), which was isolated from a patient at Addenbrooke's Hospital. Gammaglobulin containing the active antibody was conjugated to fluorescein isothiocyanate at Wellcome Research Laboratories. Work at Wellcome Research Laboratories (Fernie, personal communication) and in our own laboratory has shown that all strains of $C$ difficile so far tested have a common "O" antigen which will stain with this fluorescent antiserum.

Tissues were processed in paraffin wax and sections were cut at $5 \mu \mathrm{m}$. Dewaxed sections were rinsed in phosphate buffered saline and stained for $30 \mathrm{~min}$ at $37^{\circ} \mathrm{C}$ in antiserum diluted $1 / 5$ with phosphate buffered saline, rinsed, and mounted in phosphate buffered glycerol. They were examined with a Leitz Orthoplan microscope using a KP490 exciter and $\mathrm{K} 5 / 5$ barrier filter.

The simplified alcohol shock method, which selects for clostridial spores, ${ }^{21}$ was used for the detection of $C$ septicum from gut contents. A $1 / 10$ dilution of the specimen was added to an equal volume of absolute ethanol and left at room temperature for $1 \mathrm{~h}$. Serial 10 fold dilutions were made in brain-heart infusion broth, plated on blood agar, and incubated for $48 \mathrm{~h}$ at $37^{\circ} \mathrm{C}$ in anaerobic jars. Cultures were identified by sugar fermentation tests and gas-liquid chromatography.

\section{Case reports}

CASE 1

A 67 year old woman presented with acute myeloid leukaemia, which was treated with four courses of DAT (daunorubicin, an anthracycline in combination with cytosine arabinoside, and 6 thioguanine) over the next three months. During this period she had several episodes of neutropenia, and at the end of the second course of DAT she became febrile and intravenous vancomycin and ceftazidime were started. Griping abdominal pain and diarrhoea developed and $C$ difficile colitis was suspected (and subsequently confirmed when both the toxin and the organism were found in the stool). Oral vancomycin was added to the regimen. Ceftazidime was withdrawn when a group D streptococcus was subsequently grown from the blood. Diarrhoea and abdominal pain persisted for two weeks despite the oral vancomycin.

Bone marrow remission of the leukaemia was achieved at the end of the second month of treatment. Two months later she was readmitted to hospital, three weeks after a maintenance course of cytotoxic treatment. She complained of anorexia for five days and a one day history of abdominal pain, vomiting, fever, and constipation. She was febrile, pale, and sweaty with generalised guarding, rigidity, and rebound tenderness of the abdomen. Bowel sounds were diminished. The white blood cell count was $2.2 \times 10^{9} / 1$, with neutrophils less than $0.5 \times$ $10 \%$.

Her condition deteriorated over the next few hours. A recurrence of $C$ difficile infection complicated by colonic performation was suspected, although she had not received any antibiotics during the previous month. At laparotomy free blood stained fluid was found in the peritoneal cavity, apparently emanating from an abnormal ileocaecal region, which was excised. Perioperatively she was given intravenous fluids, cefotaxime, gentamicin, and metronidazole, but she remained hypotensive and anuric and died the next day. Blood taken on admission to hospital subsequently yielded a pure culture of $C$ septicum. $C$ difficile was not found in the bowel contents.

\section{Pathology}

The resected bowel consisted of $20 \mathrm{~cm}$ of terminal ileum together with the right colon. On both sides of the ileocaecal valve the bowel mucosa was oedematous with bulging mucosal folds and focal haemorrhage. Several small ulcers (about $0.5 \mathrm{~cm}$ diameter) were also present in the terminal ileum. Sections showed that each of the ileal ulcers was related to a mucosal leukaemic deposit associated 


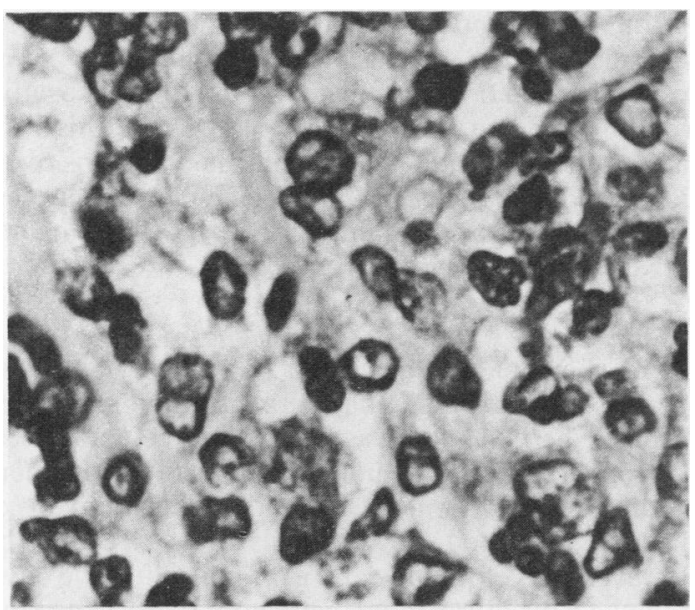

Fig. 1 Case 1. Leukaemic cells in the mucosa of the terminal ileum. Haematoxylin and eosin $\times 800$.

with areas of coagulative necrosis of both mucosa and submucosa (Fig. 1). The muscularis propria showed patchy necrosis, mainly of the inner coat. No mature polymorphonuclear leukocytes were seen, but leukaemic cells were rather sparsely scattered through the oedematous submucosa. Submucosal vessels were dilated and some contained thrombi adjacent to the areas of necrosis. Many Gram posi- tive bacilli were seen especially in the areas of necrosis, and these were all shown to be $C$ septicum by specific immunofluorescence. No $C$ difficile organisms were found. There were no leukaemic deposits in the caecum, but there were a few foci of mucosal necrosis and submucosal oedema was much more pronounced than in the ileum.

\section{CASE 2}

A 20 year old man presented with acute myeloid leukaemia and treatment with DAT was started. Two days after completing the second course he was readmitted to hospital with slight fever, abdominal pain, and diarrhoea. He was tender to palpation in the right iliac fossa. The white blood cell count was $0.4 \times 10^{\%} / 1$ with no neutrophils, and the platelet count was also reduced $\left(19 \times 10^{9} / \mathrm{l}\right)$. C difficile infection was suspected, although he had not received antibiotics during the preceding three weeks, and a course of oral vancomycin was started.

During the next $24 \mathrm{~h}$ his condition deteriorated and guarding developed in the right iliac fossa. Intravenous gentamicin, cefotaxime, and metronidazole were started before diagnostic laparotomy. At operation the caecum and ascending colon were found to be grossly thickened and haemorrhagic, and there was a perforation in the lateral wall of the colon. A right hemicolectomy was performed.

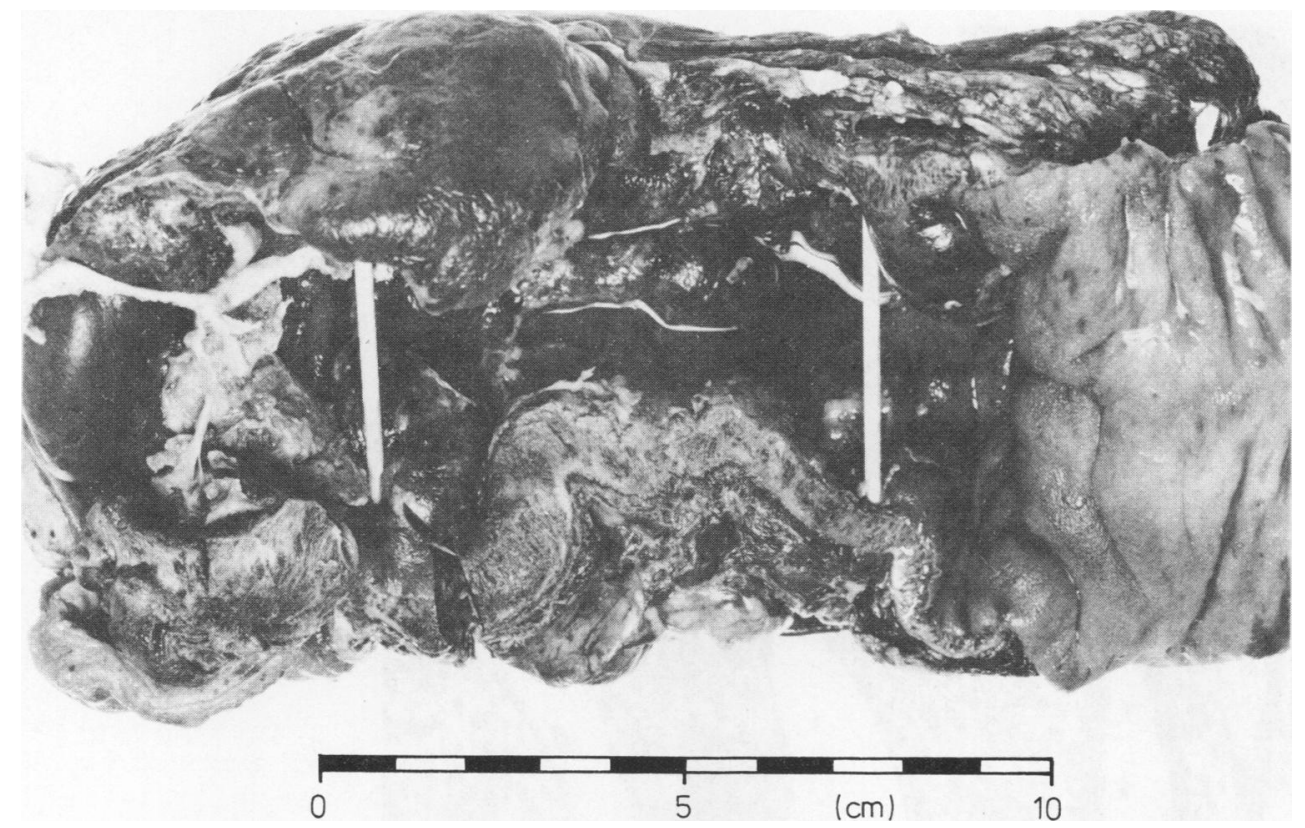

Fig. 2 Case 2. Resected caecum showing central ulceration plus gross thickening of the wall and mucosal oedema. 
His condition improved, but a blood culture taken on admission yielded clostridium species on the first postoperative day and intravenous vancomycin was added to the antibiotic regimen. The clostridium was identified as $C$ septicum and this organism was also cultured from the gut contents, but neither $C$ difficile nor its toxin was detected. The patient made an uneventful recovery.

\section{Pathology}

The surgical specimen showed a thickened purple caecum with an oedematous mucosa covered by a grey slough (Fig. 2). Sections showed striking haemorrhagic oedema of the submucosa with patchy necrosis of the mucosa and submucosa. There was no gas formation. The muscularis propria was also necrotic in some places, and at one point involvement of the full thickness of the wall had led to perforation. The intact mucosa was strikingly abnormal, the epithelium of both surface and glands having been totally replaced by atypical immature cells, which were elongated and flattened on the surface with foci of surface epithelial loss. Many gland lumina contained necrotic desquamated cells. There was some evidence of regenerative activity at the bases of the crypts (Fig. 3). In the foci of mucosal necrosis there were large numbers of organisms both in the ulcers and in the adjacent submucosa. Most organisms were Gram positive bacilli, but some were short and others filamentous; Gram negative forms were also present (Fig. 4). Despite this variable appearance all the organisms were positively identified as $C$ septicum by specific immunofluorescence (Fig. 5). None stained with the $C$ difficile antiserum.

\section{CASE 3}

A 17 year old boy in his first relapse of acute lymphoblastic leukaemia has already been described because he had what was believed to be an atypical $C$ difficile colitis. ${ }^{22}$ Remission induction treatment with DAT was followed by the development of neutropenia and fever. This was treated by intravenous cefotaxime and gentamicin for five days, followed by intravenous ceftazidine and vancomycin. On the fifth day of the second course he complained of colicky central abdominal pain and over the next $8 \mathrm{~h}$ tenderness and guarding developed in the right iliac fossa. This was accompanied by abdominal distension, scanty bowel sounds, and slight icterus. Although he had vomited several times, there was no diarrhoea.

At laparotomy the caecum and ascending colon were found to be thickened and oedematous, and so

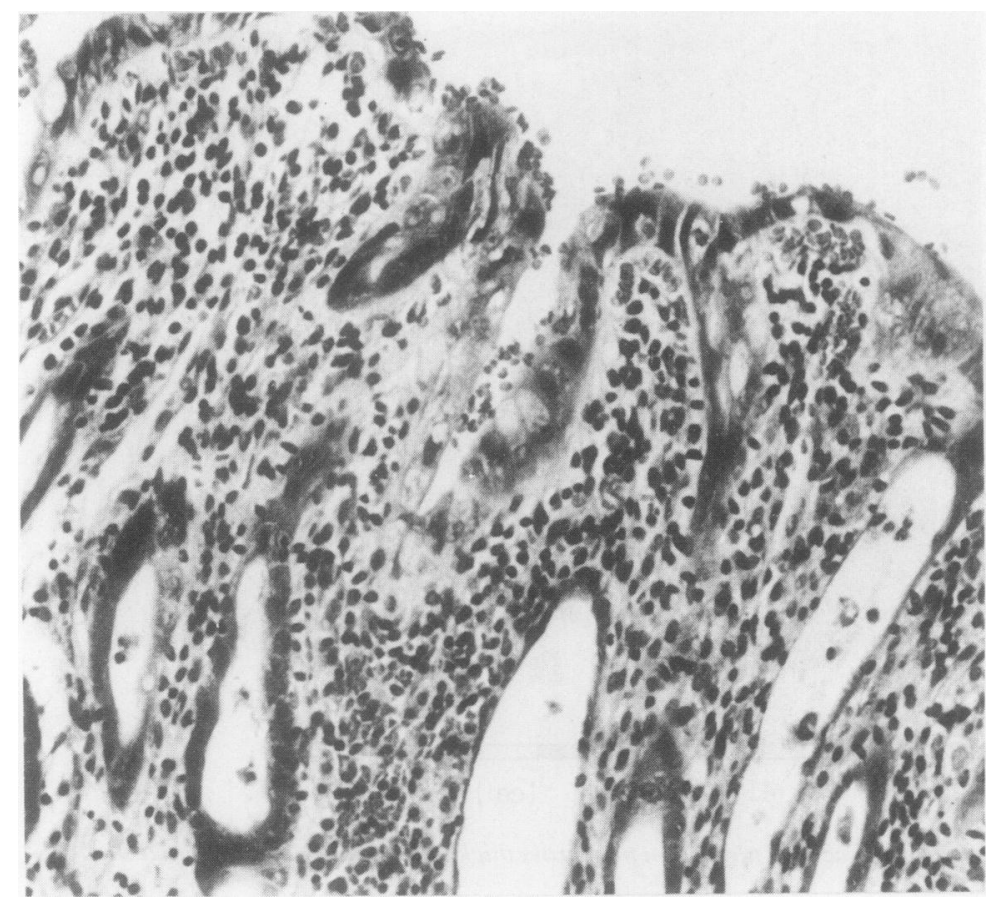

Fig. 3 Case 2. Complete replacement of the normal crypt and surface epithelium by atypical immature cells, attributable to cytotoxic drugs. Haematoxylin and eosin $\times 200$. 


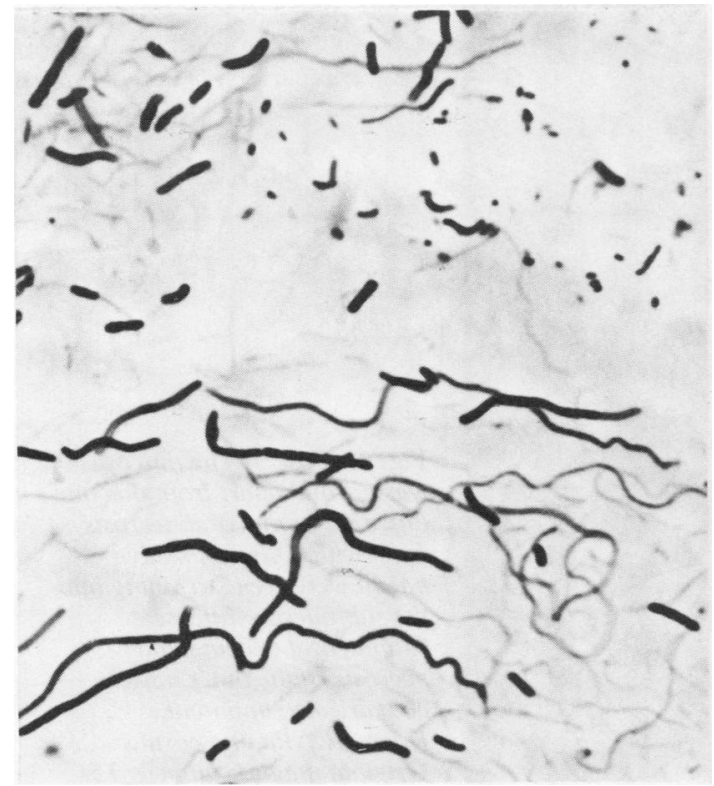

Fig. 4 Case 2. Gram stain shows the pleomorphic morphology of the C septicum organisms. Filamentous forms are intermingled with short citron forms. Most of the organisms are Gram positive but a few are negative. Gram $\times 800$.

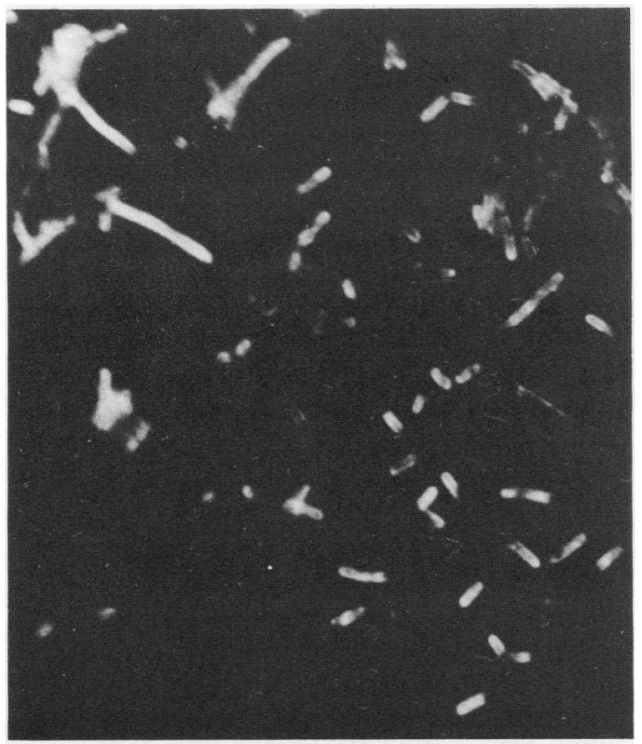

Fig. 5 Case 2. With fluorescein isothiocyanate labelled antisera to $C$ septicum all the organisms fuoresce, including the filamentous forms. $\times 800$. a right hemicolectomy was performed. $C$ difficile cytotoxin and organisms were found subsequently in caecal contents. Blood cultures were negative, but the patient was already on intravenous vancomycin at the time of operation. Although his condition initially improved and $C$ difficile disappeared from his stools after treatment with oral vancomycin, it reappeared three and a half weeks later, when he had a mild attack of diarrhoea.

\section{Pathology}

The resected colon showed a strikingly oedematous mucosa with bulging mucosal folds and focal haemorrhage but no obvious ulceration. The mesenteric vessels were patent. Sections showed intense submucosal oedema and necrosis without gas formation, which was most pronounced in the caecum but also present in the ileal stump. There was patchy necrosis of the muscularis propria, particularly of its inner layer, but the mucosa appeared intact and normal. It was only after careful search and multiple sections that a single focus of mucosal necrosis $(0 \cdot 2$ $\mathrm{cm}$ ) was discovered (Fig. 6). Multiple Gram positive bacilli could be seen streaming into the submucosa from this point. All the organisms were shown by specific immunofluorescence to be $C$ septicum. No $C$ difficile was found.

\section{Discussion}

C septicum was long thought to be important in human pathology, but in recent years it has suffered a relative eclipse. ${ }^{23}$ It is responsible for $10-20 \%$ of cases of gas gangrene, which is now a rare disease both in civilian life and under war conditions. ${ }^{24}$ In veterinary published work $C$ septicum has been called the malignant oedema bacillus because of the characteristic findings in animal infections. The affected tissues are infiltrated with large quantities of gelatinous exudate with little or no gas. ${ }^{25}$ The organism is common in the intestinal tract of herbivores and is associated with braxy in sheep, a condition with histological similarities to neutropenic enterocolitis. Before vaccines became available braxy caused high losses in Norway, Scotland, and Iceland. ${ }^{26}$ The disease follows ingestion of frozen grass which fills the rumen and chills the anatomically adjacent abomasum (fourth stomach). $C$ septicum invades the wall of the abomasum, producing enormous thickening of the wall due to inflammatory oedema, in which there are large numbers of bacilli and white cells. Mucosal haemorrhages are also seen. ${ }^{26}$

The classic descriptions of neutropenic enterocolitis and $C$ septicum infection are similar and correspond closely to the findings in our three 


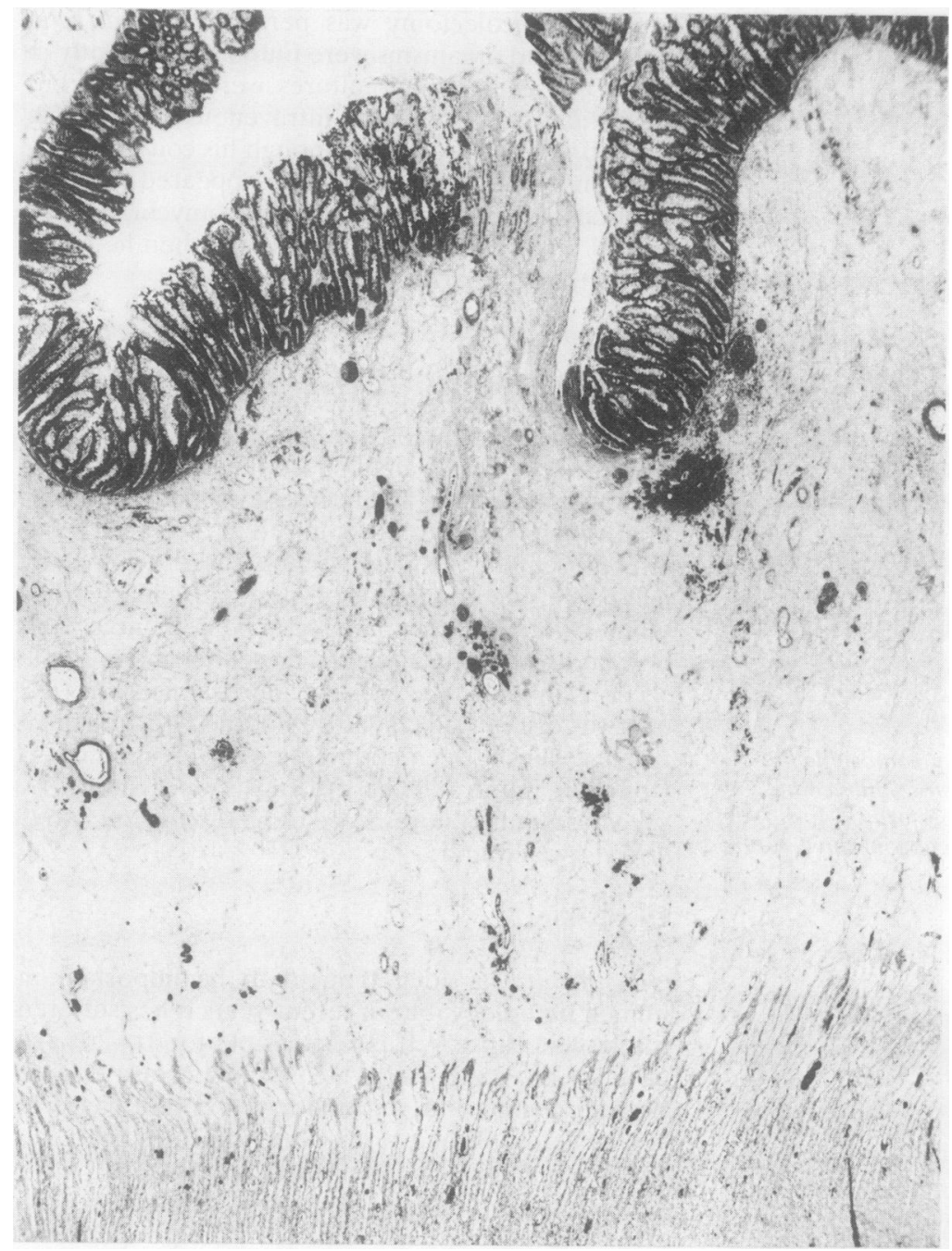

Fig. 6 Case 3. The mucosa was normal apart from this one tiny area of mucosal necrosis from which Gram positive bacilli were seen streaming into the submucosa. Intense submucosal oedema and necrosis of the inner muscle coat are also apparent. Haematoxylin and eosin. Original magnification $\times 15$.

patients. In each of the cases extensive submucosal oedema with necrosis and haemorrhage without gas formation were the main findings. Myonecrosis in the absence of vasculitis was also seen. Organisms were plentiful and always most numerous near to the surface, where the mucosa was invariably necrotic, suggesting that infection was from the lumen of the bowel. All of our patients had $C$ septicum in the gut wall as shown by immunofluorescence. $C$ septicum was cultured from the blood of two of our patients, while the third was already receiving systemic antibiotics effective against clostridia, as are many such neutropenic patients.

All the patients presented with abdominal pain and fever and, as in other cases associated with $C$ septicum septicaemia, symptoms developed rapidly over $24-48 \mathrm{~h}$. Vomiting and change in bowel habit were inconsistent findings.

There is some disagreement as to how commonly C septicum is found in the normal human intestine. Although it is often stated that it is a member of the normal flora, ${ }^{23}$ recent studies suggest that it is rare. ${ }^{27}{ }^{28}$ Gut contents were cultured for $C$ septicum only in case 2 , but there seems no doubt that this was the portal of entry in our patients. It is not clear why the ileocaecal region is so often the prime target of the infection. Bacterial proliferation at this site is usual and is probably affected by local factors such as $\mathrm{pH}$ and the redox potential, the osmotic environment, and bile salt concentration..$^{18}$ By analogy with 
Possible pathogenetic mechanisms in neutropenic enterocolitis

$C$ septicum and mucosal damage due to:

1 Leukaemic deposits (case 1)

2 Cytotoxic drugs (case 2)

3 Ulceration of tumours

4 Neutropenic ulceration

5 Haemorrhage due to thrombocytopenia

6 ?Clostridium difficile (case 3 )

$C$ difficile infection, it is likely that $C$ septicum colonisation is affected by antibiotics, and it is important to note that this organism is much more resistant to third generation cephalosporins and thienamycin than earlier cephalosporins or penicillins. ${ }^{29}$

It seems probable that, as in braxy, predisposing physiological and structural damage to the mucosal barrier is necessary to initiate invasive $C$ septicum infection (Table). Also the relative rarity of $C$ perfringens bacteraemia in neutropenic patients suggests that they may have a specific susceptibility to $C$ septicum. Our patients showed three possible mechanisms. In case 2 there was extensive epithelial atypia present in the colonic mucosa, which, as in the cases described by Slavin, ${ }^{2}$ was associated with patchy necrosis of epithelial cells leading to attenuation and interruption of the surface layer in places. These changes are attributable to cytosine arabinoside, which was received by all of our patients. Slavin et $a l^{2}$ do not indicate whether the epithelial lesions have any predisposition for the ileocaecal region, but caecal necrosis has been encountered with other cytotoxic regimens. ${ }^{30} 31$

In case 1 the lesions were clearly spatially related to ulcerated mucosal leukaemic deposits, another obvious portal of entry. This same mechanism, surface ulceration of tumours, probably accounts for the strong association between $C$ septicum and cancers of the caecum. ${ }^{17-14}$ Another possible mechanism may be mucosal damage resulting from haemorrhage due to thrombocytopenia. ${ }^{17}$

None of these mechanisms seems able to explain infection in patients with non-neoplastic agranulocytosis. Neutropenia, however, is associated with ulceration of the oral mucous membrane, ${ }^{12}$ and similar lesions occur in the caecum. ${ }^{32}$

Case 3 seems the most problematic. This case was initially reported as an atypical response to $C$ difficile in a patient without neutrophils. ${ }^{22} C$ difficile and its toxin were undoubtedly present in the stool, but we have conclusively shown by specific immunofluorescence that the organisms present in the lesions were not $C$ difficile but were in fact $C$ septicum.

$C$ difficile is now considered to be the main organism responsible for pseudomembranous enterocolitis, ${ }^{33}{ }^{34}$ which is usually associated with alteration to the normal gut bacterial flora caused by antibiotics. ${ }^{34}$ Morphologically, in non-neutropenic patients, the changes are distinctive..$^{35}$ The colonic mucosa is covered by raised yellowish plaques or pseudomembranes, which have no special predilection for the caecum. Microscopically, the plaques are composed of foci of mucosal necrosis covered by a volcano-like eruption of neutrophils, fibrin, and epithelial debris. True ulceration or involvement of the submucosa are not normally seen. Bacteria are not found in the lesions.

Since 1975 the hamster has been widely adopted as an experimental model of pseudomembranous enterocolitis since it has been shown that a haemorrhagic caecitis can be reproduced by $C$ difficile or its toxin..$^{36}$ Although this anatomical setting suggests a close parallel with neutropenic enterocolitis, morphologically the lesions are dissimilar. Microscopically the main changes are seen in the mucosa, which appears hyperplastic with diffuse haemorrhage. ${ }^{38}{ }^{39}$ In the most advanced cases there is extensive epithelial necrosis with the formation of pseudomembranes. Organisms have not been found within the tissues. Furthermore, it has been conclusively shown that the lesions can be reproduced by intracaecal injection of the toxin alone. ${ }^{36}$ Here too the principal histological change is epithelial necrosis.

Case 3 showed none of the usual features of pseudomembranous enterocolitis, and we remain ignorant of the usual morphological consequences of infection by $C$ difficile in neutropenia. It remains to be shown that $C$ difficile has the power to cause tissue damage in the bowel wall by invasion. It is probable that this organism was responsible for the trivial mucosal damage in case 3, allowing invasion by the more aggressive $C$ septicum. The attractive hypothesis that neutropenic enterocolitis might have been directly a manifestation of $C$ difficile infection alone $^{2240}$ is thus no longer tenable in this particular case, and a synergic process entailing two clostridial species seems more likely.

Other clostridia are well recognised as causes of gut necrosis in animals, and $C$ perfringens type $\mathrm{C}$ causes enteritis necroticans in man. The infection is histologically different from the cases we describe. As well as tending to occur in the upper gastrointestinal tract, the findings are dominated by haemorrhagic necrosis and submucosal gas bubbles rather than oedema, as well as systemic effects such as disseminated intravascular coagulation.

Previous reports of neutropenic enterocolitis have emphasised that multiple organisms are often found. ${ }^{347}$ It is interesting that many of these organisms - for example, pseudomonas, aeromonas, 
and aspergillus - may be associated with vascular invasion and might thus be responsible for mucosal damage predisposing to clostridial invasion.

There are other explanations for the mixed bacterial flora seen on Gram stained slides. Firstly, $C$ septicum is a highly pleomorphic organism which may range in size from long filaments to short citron and coccobacillary forms. Furthermore, as in old cultures, many of the organisms in lesions appear Gram negative, giving an impression that more than one organism is present. Secondly, as Blenkinsopp and Dupont ${ }^{41}$ indicate, invasion of extensively necrotic gut wall by bowel flora may mask a primarily clostridial disease. This could also mask the responsible organism in postmortem bacteriology.? Since all our cases were surgical excisions there had been little or no opportunity for overgrowth by other bacterial species.

Our experience with these three patients suggests that it may be valuable to screen neutropenic patients with abdominal symptoms for gut colonisation by $C$ septicum as well as $C$ difficile. The alcohol shock method using non-selective nutrient blood agar is an ideal and simple method. This may be particularly valuable in patients receiving antibiotics that prevent detection of bacteraemia as the spores will not be affected by the antibiotic. No model for clostridial infection in neutropenic animals has been devised. In neutropenic patients many species of clostridia may be found in the blood as components of polymicrobial bacteraemia. By contrast $C$ septicum is usually isolated in pure growth, suggesting a primary pathogenic role. Other clostridia may also have this potential. The role of $C$ difficile in neutropenic enterocolitis remains controversial. We have recovered two isolates as components of polymicrobial bacteraemia which could suggest either a potential for tissue invasion or a polymicrobial bacteraemia associated with colonic ulceration (Rampling et al. Unpublished observations). In either case it is likely that oral vancomycin and parenteral antibiotics effective against anaerobes will be appropriate treatment, as they would also be in $C$ septicum infection.

In conclusion, the lesions of neutropenic enterocolitis have many of the features of the classic descriptions of infection by $C$ septicum. Others have found the organism in the blood-we have found it in the bowel wall. This observation, made on three cases occurring in one unit in nine months, provides further support for Rifkin's suggestion ${ }^{13}$ that this organism may play an important primary role in many more cases of neutropenic colitis than hitherto appreciated. The epidemiology of $C$ septicum thus deserves further study, and active immunisation of patients at risk may well be justified in the future.
We are most grateful to Dr David Fernie of Wellcome Research Laboratories, who kindly arranged for the preparation of the fluorescent antibody to $C$ difficile and who donated the $C$ septicum fluorescent antibody for this investigation. We are also indebted to $\mathrm{Mr}$ AJ Newton, who performed the immunocytochemical methods, and to Mr Christopher Burton for the photographs.

\section{References}

' Prolla JC, Kirsner JB. The gastrointestinal lesions and complications of the leukaemias. Ann Intern Med 1964;61:1084-103.

${ }^{2}$ Slavin RE, Dias MA, Saral R. Cytosine arabinoside induced gastrointestinal toxic alterations in sequential chemotherapeutic protocols. A clinicopathologic study of 33 patients. Cancer 1978;42: 1747-59.

${ }^{3}$ Steinberg D, Gold J, Brodin A. Necrotizing enterocolitis in leukaemia. Arch Intern Med 1973;131:538-44.

${ }^{4}$ Dosik GM, Luna M, Valdivieso M, et al. Necrotizing colitis in patients with cancer. Am J Med 1979;67:646-56.

s Wagner ML, Rosenberg HS, Fernbach DJ, Singleton EB. Typhlitis: A complication of leukaemia in childhood. $A J R$ 1970; 109:341-50.

- Sherman NJ, Woolley MM. The ileocaecal syndrome in acute childhood leukaemia. Arch Surg 1973;107:39-42.

7 Moir DH, Bale PM. Necropsy findings in childhood leukaemia, emphasizing neutropenic enterocolitis and cerebral calcification. Pathology 1976;8:247-58.

${ }^{8}$ Kies MS, Luedke DW, Boyd JF, McCue MJ. Neutropenic enterocolitis. Two case reports of long-term survival following surgery. Cancer 1979;43:730-4.

' Varki AP, Armitage JO, Feagler JR. Typhlitis in acute leukaemia. Successful treatment by early surgical intervention. Cancer 1979;43:695-7.

${ }^{10}$ Ikard RW. Neutropenic typhlitis in adults. Arch Surg 1981;116: 943-5.

"Shaked A, Shinar E, Freund H. Neutropenic typhlitis: a plea for conservation. Dis Colon Rectum 1983;26:351-2.

${ }_{12}$ Bignold LP, Harvey HPB. Necrotizing enterocolitis associated with invasion by Clostridium septicum complicating cyclic neutropenia. Aust NZ J Med 1979;9:426-9.

${ }^{13}$ Rifkin GD. Neutropenic enterocolitis and Clostridium septicum infection in patients with agranulocytosis. Arch Intern Med 1980;140:834-5.

14 Hopkins DG, Kushner JP. Clostridial species in the pathogenesis of necrotizing enterocolitis in patients with neutropenia. Am J Hematol 1983;14:289-94.

15 Wright DG, Dale DC, Fauci AS; :Wolff SM. Human cyclic neutropenia. Clinical review and long-term follow-up of patients. Medicine (Balt) 1981;60:1-13.

${ }^{16}$ Braye SG, Copplestone JA, Gartell PC. Neutropenic enterocolitis during mianserin-induced agranulocytosis. $\mathrm{Br}$ Med J 1982;285: 1117 .

17 Alpern RJ, Dowell VR. Clostridium septicum infections and malignancy. JAMA 1969;209:385-8.

${ }^{18}$ Koransky JR, Stargel MD, Dowell VR. Clostridium septicum bacteraemia. Its clinical significance. Am J Med 1979;66:63-6.

${ }^{19}$ Katlic MR, Derkac WM, Coleman WS. Clostridium septicum infection and malignancy. Ann Surg 1981;193:361-4.

${ }^{20}$ Moussa RS. Antigenic formulae for Clostridium septicum and Clostridium chauvei. J Pathol Bacteriol 1959;77:341-50.

${ }^{21}$ Borriello SP, Honour P. Simplified procedure for the routine isolation of Clostridium difficile from faeces. J Clin Pathol 1981;34:1124-7.

${ }^{22}$ Rampling A, Warren RE, Berry PJ, Swirsky D, Hoggarth C, Bevan PC. Atypical Clostridium difficile colitis in neutropenic 
patients. Lancet 1982;ii:162-3.

${ }^{23}$ MacLennan JD. The histotoxic clostridial infections of man. Bacteriol Rev 1962;26:236-76.

${ }^{24}$ Finegold SM. Anaerobic bacteria in human disease. New York: Academic Press, 1977:388-9.

${ }^{25}$ Topley WWC, Wilson GS. Gas gangrene; Anaerobic infections of animals. The principles of bacteriology and immunity. London: Edward Arnold and Co, 1936:1403-8.

${ }^{20}$ Gaiger SH. Anaerobic infections of animals. J Comp Pathol 1924;37:163-91.

${ }^{27}$ Drasar BS, Goddard P, Heaton S. Clostridia isolated from faeces. J Med Microbiol 1976;9:63-71.

${ }^{28}$ Finegold SM, Atterbery HR, Sutter VL. Effect of diet on human fecal flora: comparison of Japanese and American diets. Am J Clin Nutr 1974;27: 1456-69.

${ }^{29}$ Gabay EL, Rolfe RD, Finegold SM. Susceptibility of Clostridium septicum to 23 antimicrobial agents. Antimicrob Agents Chemother 1981;20:852-3.

${ }^{30}$ Matolo NM, Garfinkle SE, Wolfman EF. Intestinal necrosis and perforation in patients receiving immunosuppressive drugs. Am J Surg 1976;132:753-4.

${ }^{31}$ Kingry RL, Hobson RW, Muir RW. Caecal necrosis and perforation with systemic chemotherapy. Am Surg 1973;39:129-33.

${ }^{32}$ Geelhoed GW, Kane MA, Dale DC, Wells SA. Colon ulceration and perforation in cyclic neutropenia. J Pediatr Surg 1973;8:379-82.

${ }^{33}$ Tedesco FJ. Pseudomembranous colitis: Pathogenesis and therapy. Med Clin North Am 1982;66:655-64.
${ }^{34}$ O' Connor TW. Pseudomembranous enterocolitis: A historical and clinical view. Dis Colon Rectum 1981;24:445-8.

${ }^{35}$ Bartlett JG. Antibiotic-associated pseudomembranous colitis. Rev Infect Dis 1979;1:530-9.

${ }^{36}$ Libby JM, Jortner BS, Wilkins TD. Effects of the two toxins of Clostridium difficile in antibiotic-associated cecitis in hamsters. Infect Immun 1982;36:882-9.

${ }^{37}$ Lusk RH, Fekety R, Silva J, Browne RA, Ringler DH, Abrams GD. Clindamycin-induced enterocolitis in hamsters. J Infect Dis 1978;137:464-75.

${ }^{38}$ Humphrey CD, Lushbaugh WB, Condon CW, Pittman JC, Pittman FE. Light and electron microscopic studies of antibiotic associated colitis in the hamster. Gut 1979;20:6-15.

${ }^{39}$ Price AB, Larson HE, Crow J. Morphology of experimental antibiotic-associated enterocolitis in the hamster: a model for human pseudomembranous colitis and antibiotic associated diarrhoea. Gut 1979;20:467-75.

${ }^{40}$ Conzigou P, Reiffers J, Levêque AM, Amouretti M, Broustet A, Berand C. Les entérocolites aiguës nécrosantes des agranulocytoses sout des formes majeures de colites pseudomembraneuses. Gastroenterol Clin Biol 1982;6:48-51.

${ }^{41}$ Blenkinsopp WK, Dupont PA. Bacteria in necrotising enterocolitis. Lancet 1977;ii:617.

Requests for reprints to: Dr DGD Wight, Department of Histopathology. Addenbrooke's Hospital, Cambridge CB2 2QQ. England. 